\title{
A mosaic form of microphthalmia with linear skin defects
}

\author{
Nina Prepeluh¹, Bojan Korpar², Andreja Zagorac', Boris Zagradišnik', Andreja Golub and Nadja Kokalj Vokač ${ }^{1,3^{*}}$
}

\begin{abstract}
Background: Microphthalmia with linear skin defects (MLS) syndrome is a rare neurodevelopmental X-dominant disorder. It presents in females as it is normally lethal in males. Three causative genes for MLS syndrome (OMIM 309801) have been identified all taking part in mitochondrial respiratory chain and oxidative phosphorylation. In our case, we describe a newborn with mosaic deletion encompassing HCCS gene resulting in unilateral microphthalmia and facial skin lesions.

Case presentation: A girl was born with caesarean section at 40 weeks of gestation. Clinical findings revealed anophthalmia of the left eye. The left eyelids were intact, the orbit was empty and the right eye was normal, without any abnormalities. She had typical linear skin defects on the left cheek, one on the left side of the neck, and two on the 3th and 4th fingers of the left hand. The other clinical findings and the neurological exam were normal. US of the brain and EEG were normal. Molecular karyotyping using BlueGnome CytoChip Oligo 4× 180K array was performed detecting an approximately 18\% mosaic $3.3 \mathrm{Mb}$ deletion (arr[GRCh37] Xp22.31 p22.2(8,622,553_11,887,361)× 1[0.18]). FISH using RPCI1 1-768H20 BAC clone on cultivated interphase and metaphase lymphocytes was used to confirm the array results. The observed deletion was present in 29\% of cells (46,XX,ish del(p22.2p22.31)(RPCl11-768H20)[60/205]).

Conclusions: In this report we present a female proband with MLS syndrome. To our knowledge, there have been only few other cases of mosaic MLS syndrome described in the literature. Our case shows that low grade mosaicism does not preclude full clinical presentation and further supports the critical role of the X inactivation pattern in the development of the clinical findings.
\end{abstract}

Keywords: Microphthalmia, Linear skin defects, Mosaicism, Xp22.31p22.2 deletion, HSSC gene

\section{Background}

Microphthalmia with linear skin defects (MLS) syndrome is a rare $\mathrm{X}$-linked dominant disorder affecting mainly the eyes and the skin. It affects only girls as it is lethal in males. The characteristic features of the syndrome are microphthalmia (sometimes anophthalmia) that may be uni- or bilateral and linear skin defects, which are usually limited to the face and neck. The accompanying abnormalities include other ocular abnormalities, structural neurological abnormalities, developmental delay, congenital heart defects and short stature. The disorder shows high phenotypic variability among affected individuals including between family members $[1-3]$.

\footnotetext{
* Correspondence: nadja.kokalj-vokac@ukc-mb.si

'Laboratory of Medical Genetics, University Medical Centre Maribor Ljubljanska 5, 2000 Maribor, Slovenia

${ }^{3}$ Medical Faculty, University of Maribor, Maribor, Slovenia

Full list of author information is available at the end of the article
}

To date, less than a hundred patients with MLS syndrome have been reported. The syndrome is most often a result of a de novo pathogenic mutation, although rare familial cases have been described [3-5]. Genetic abnormalities resulting in Xp22.2 monosomy as well as some intragenic deletions and point mutations in $H C C S$ and $C O X 7 B$ genes were found to be responsible for the syndrome. Recently a mutation in NDUFB11 gene was reported as causative in two cases. All three genes encode proteins that are essential for proper function of mitochondrial respiratory chain (MRC) and oxidative phosphorylation [6-8]. Therefore, it is possible that the impaired oxidative phosphorylation and its consequences (e.g. lack of ATP, abnormal production of free oxygen radicals) are responsible for the clinical manifestations observed in MLS syndrome [8].

In this report we present the clinical and molecular data of an infant girl with mosaic deletion of the chromosome Xp22.2. The deletion encompasses 12 
OMIM genes, including HCCS gene, and is present in approximately $18 \%$ of the cells. Despite the mosaic form of MLS syndrome, the girl presents with the fully expressed clinical presentation.

\section{Case presentation}

The female proband is the first child of healthy parents. None of the parents showed any dysmorphic signs and their family history was unremarkable. An infant girl was born with elective caesarean section at 40 weeks of gestation. Birth weight was $3640 \mathrm{~g}$, birth length $50 \mathrm{~cm}$, head circumference $35 \mathrm{~cm}$ and the Apgar scores were 9/ 9/9. Mother had gestational diabetes mellitus controlled by diet and was treated with beta blockers in the last month of pregnancy because of paroxysmal tachycardia.

At birth the newborn female showed linear skin defects on the left cheek, one on the left side of the neck and two on the 3th and 4th fingers of the left hand. Ophthalmologic evaluation revealed left-sided anophthalmia with intact left eyelids (Fig.1). The right eye was without any abnormalities. Echocardiography detected atrial septal defect and patent ductus arteriosus, which was not hemodynamically important. Other clinical findings and neurological exam were normal. Brain US and EEG were also normal.

The clinical diagnosis of MLS syndrome was suspected and a blood sample was taken for microarray analysis.

At the follow-up two months later, the skin defects were almost completely healed and only barely seen.

\section{Genetic analysis}

Molecular karyotyping using the BlueGnome CytoChip Oligo $4 \times 180 \mathrm{~K}$ array was performed following the protocol. The array $\mathrm{CGH}$ analysis demonstrated an approximately $18 \%$ mosaic $3.3 \mathrm{Mb}$ deletion of Xp22.31p22.2 (arr [GRCh37] Xp22.31p22.2(8622553-11,887,361) × 1[0.18]) (Fig.2). The deletion encompasses 12 OMIM genes, including KAL1, FAM9A, FAM9B, TBL1X, GPR143, SHROOM2, CLCN4, MID1, HCCS, ARHGAP6, AMELX and MSL3.

To confirm the array results, FISH using BAC probe RPCI11-768H20 on cultivated lymphocytes was used. Mosaicism was confirmed in $29 \%$ of cells by producing only one hybridisation signal (of the normal chromosome X) instead of two (Fig.3).

$\mathrm{X}$ chromosome inactivation $(\mathrm{XCI})$ pattern was performed by examining the methylation status of the AR1 and AR4 loci using a standard protocol. A ratio 92:8 $\mathrm{XCI}$ was detected.

\section{Discussion and conclusion}

In this paper we report an infant girl with a mosaic interstitial Xp22.2 deletion. A newborn presented with typical clinical signs of MLS syndrome - congenital

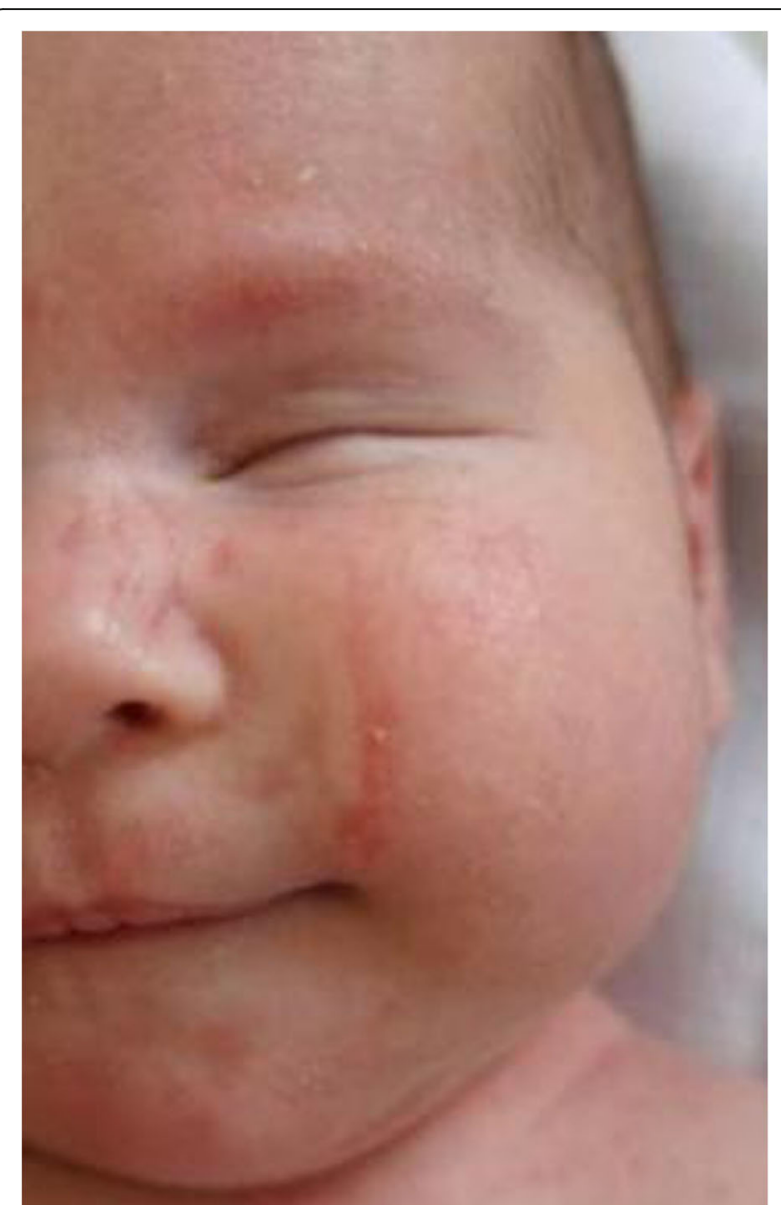

Fig. 1 An infant girl with left-sided anophthalmia and skin defect on the cheek

linear skin defects and left-sided anophthalmia, accompanied by congenital heart defects. The latter is known to be an associated feature of the syndrome and represents one of the so called "minor criteria of MLS syndrome".

The syndrome was firstly reported to be caused by genetic abnormalities resulting in Xp22.2 monosomy. With technological advances we detected pathogenic mutations in three critical genes encoding proteins important in mitochondrial respiratory chain and oxidative phosphorylation - HCCS, COX7B and NDUFB11 genes. Although the exact pathophysiological mechanism of disease is not known yet, it is hypothesised that cells with the mutated $\mathrm{X}$ chromosome active express impaired oxidative phosphorylation which leads to cell dying and development of MLS- associated features [8, 9].

The disorder may present with a broad spectrum of congenital anomalies. The majority of patients show classical eye and skin abnormalities, but the clinical presentation may vary widely across patients and even within the family members $[1-4,10,11]$. Van Rahden 


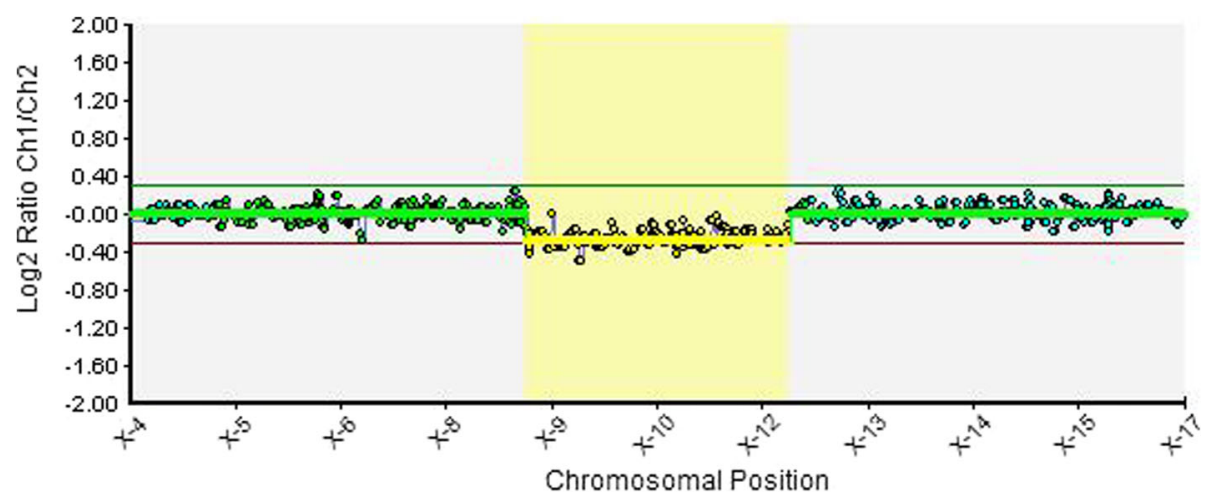

Fig. 2 Array CGH of the patient revealed deletion of Xp22.2 in mosaic form: $\operatorname{arr}[G R C h 37] X p 22.31$ p22.2(8622553-11,887,361)× [0.18]

et al. reported of six patients who all presented with microphthalmia, two had accompanied anophthalmia and five had sclerocornea. Linear skin defects were found in four patients while were other abnormalities limited to single cases [3]. Wimplinger et al. described a familial form of MLS syndrome showing high clinical variability between patients. They reported of a mother and two daughters who had intragenic deletion in HCCS gene. While mother was asymptomatic and one daughter showed only ocular abnormalities, the youngest one presented with left-sided anophthalmia, sclerocornea and skin defect [2]. Interestingly, although no clear genotype-phenotype correlations have been made so far, it seems that there are some variations in clinical picture according to the mutated gene $[3,10,12]$. Indrieri et al. described four patients with MLS syndrome caused by $C O X 7 B$ mutations, who show only linear skin defects and lack of ocular involvement. This is in contrast to HCCS mutations which normally affect both the eye and skin as is seen in our case [7].
The mechanism responsible for high phenotypic variability observed is currently unknown, although $\mathrm{X}$ inactivation patterns and presence of mosaicism are thought to play important role $[2-4,9,13]$. Cells with active abnormal $\mathrm{X}$ chromosome are thought to die earlier which leads to the predominance of the cells with active wild-type X chromosome, skewed XCI and milder phenotype $[3,13]$. To date only few cases of mosaic form of MLS syndrome have been described. Wimplinger et al. reported a girl with classical signs of MLS syndrome and her asymptomatic mother who presented only with short stature and low posterior hairline. It was found that both carry terminal Xp deletion (mother in mosaic form - 45,X $[11] / 46, \operatorname{del}(\mathrm{X})(\mathrm{p} 22.2)[89])$ and have a completely skewed $\mathrm{X}$ inactivation. It was concluded that both mechanisms the somatic mosaicism and non-random $\mathrm{X}$ - inactivation pattern in favour of non-mutated $\mathrm{X}$ chromosome might contribute to the lack of symptoms in the mother [9]. On the contrary, Ogata et al. reported a girl with a complex karyotype and a deletion of Xp22 presented in $58 \%$ of

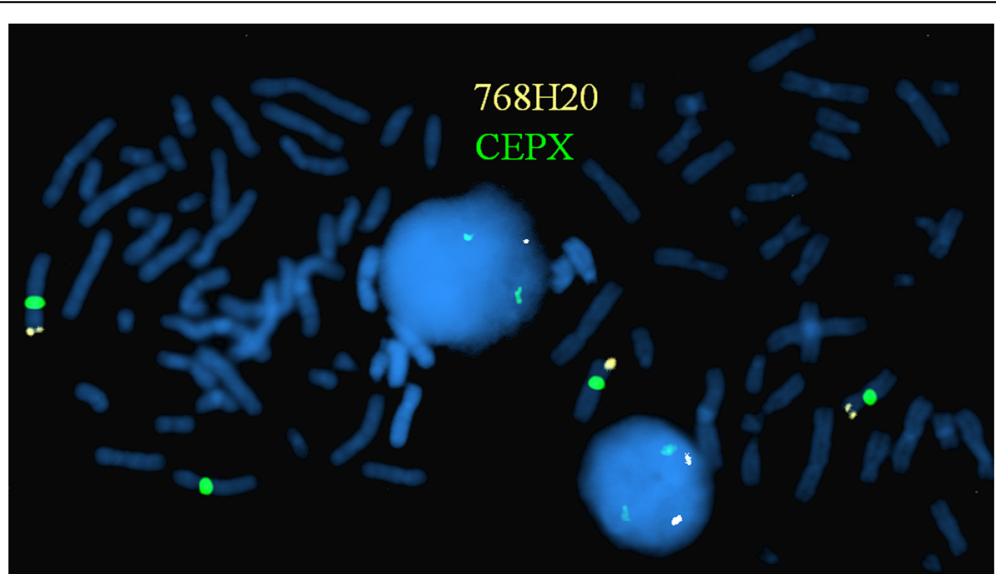

Fig. 3 FISH analysis with RPCI11-768H20 (gold) and CEPX (green) DNA probes on double metaphase spreads and interphase nuclei. One metaphase and one nuclei with deletion of RPCI1 1-768 20 probe on $\mathrm{X}$ chromosome and another with two normal $\mathrm{X}$ chromosomes and two signals for RPCI11-768H20 probe, demonstrating mosaicism 
cells. She presented with right-sided microphthalmia, sclerocornea, skin defects and absent corpus callosum. The full blown clinical presentation was hypothesised to be the consequence of inactivation of the normal $\mathrm{X}$ chromosome [14]. Similarly, our proband girl presented with fully expressed clinical symptoms despite low grade mosaicism and apparently skewed XCI detected in her blood cells. One possible explanation for the clinical presentation observed is that we tested only blood cells so the percent of cells with a deletion of Xp22 among other tissues is not known. It is possible that the percent of the mutated cells among blood cells is under-represented due to a negative selection. Furthermore, taking into consideration that the mutation was presented only in $18-29 \%$ of cells, we interpret the detected XCI ratio 92:8 in blood cells as partially by chance, which also contribute to the explanation the clinical presentation. The XCI pattern in other tissues is however not known. Taking together, we think that partially random $\mathrm{X}$ inactivation pattern and the possibility of the higher percent of cells with the deletion of Xp22 among critical tissues play the pivotal role in developing the full-blown clinical picture observed in our case.

To sum up, in this report we present an infant girl with mosaic deletion of Xp22.2 and a fully expressed MLS syndrome despite low grade mosaicism detected. To our knowledge, this case presents with the lowest mosaicism reported in association with MLS syndrome. It shows that the mosaicism does not preclude the full clinical expression and supports the critical role of $\mathrm{X}$ inactivation pattern in the development of clinical presentation.

\section{Abbreviations}

ATP: Adenosine triphosphate; BAC: Bacterial artificial chromosome; CGH: Comparative genomic hybridization; EEG: Electroencephalogram; FISH: Fluorescent in situ hybridization; GRCh37: Genome Reference Consortium human genome (build 37); MLS: Microphthalmia with linear skin defects syndrome; OMIM: Online Mendelian Inheritance in Man; RPCl: Bacterial Artificial Chromosome Library; US: Ultrasound; XCl: X chromosome inactivation

\section{Acknowledgements}

We are thankful to the patients of the infant girl for allowing us to publish this paper.

We declar that abstract has been previously published. Namely as, "Meeting abstracts from the 11th European Cytogenetics Conference." Molecular Cytogenetics 2017, 10(suppl 1):20. DOl https://doi.org/10.1186/s13039-017-0319-3.

\section{Funding}

This work was supported by the Ministry of Science and Technology, Slovenia grant no. P04-220 Research Program.

\section{Availability of data and materials}

All data generated or analyzed during this study are included in this published article. Data can also be found in Decipher database, Case No.: 343910 (https://decipher.sanger.ac.uk/)

\section{Authors' contributions}

NP wrote the article, BK cared for the patient, collected samples, AZ performed and analyzed the array results, and BZ performed $X$ inactivation study, AG performed genetic counselling in patient family, NKV designed the study and critically read the revised manuscript. All authors read and approved the final manuscript.

\section{Ethics approval and consent to participate}

This study was performed in accordance with the Declaration of Helsinki, after written informed consent obtained from the legal guardians. Our institution does not consider a case report as human subject research and this falls in the realm of routine clinical care. As a result, an ethical approval is not required for a case report.

\section{Consent for publication}

Written informed consent was obtained from the participants or legal guardians for publication of this case report and accompanying images. A copy of the written consent is available for review by the Editor of BMC Pediatrics.

\section{Competing interests}

The authors declare that they have no competing interests.

\section{Publisher's Note}

Springer Nature remains neutral with regard to jurisdictional claims in published maps and institutional affiliations.

\section{Author details}

'Laboratory of Medical Genetics, University Medical Centre Maribor, Ljubljanska 5, 2000 Maribor, Slovenia. ${ }^{2}$ Department of Perinatology, Division of Gynaecology and Perinatology, University Medical Centre Maribor, Maribor, Slovenia. ${ }^{3}$ Medical Faculty, University of Maribor, Maribor, Slovenia.

Received: 29 August 2017 Accepted: 20 July 2018

Published online: 01 August 2018

\section{References}

1. Lindsay EA, Grillo A, Ferrero GB, et al. Microphthalmia with linear skin defects (MLS) syndrome: clinical, cytogenetic, and molecular characterization. Am J Med Genet. 1994;49:229-34.

2. Wimplinger I, Morleo M, Rosenberger $\mathrm{G}$, et al. Mutations of the mitochondrial holocytochrome c-type synthase in X-linked dominant microphthalmia with linear skin defects syndrome. Am J Hum Genet. 2006; 79:878-89.

3. Van rahden VA, Rau I, Fuchs S, et al. Clinical spectrum of females with HCCS mutation: from no clinical signs to a neonatal lethal form of the microphthalmia with linear skin defects (MLS) syndrome. Orphanet J Rare Dis. 2014;9:53.

4. Vergult S, Leroy B, Claerhout I, Menten B. Familial cases of a submicroscopic Xp22.2 deletion: genotype-phenotype correlation in microphthalmia with linear skin defects syndrome. Mol Vis. 2013;19:311-8.

5. Margari L, Colonna A, Craig F, et al. Microphthalmia with Linear Skin Defects (MLS) associated with Autism Spectrum Disorder (ASD) in a patient with Familial 12.9Mb Terminal Xp deletion. BMC Pediatr. 2014;14:220.

6. Jiang X, Wang X. Cytochrome C-mediated apoptosis. Annu Rev Biochem. 2004;73:87-106.

7. Indrieri A, Van rahden VA, Tiranti V, et al. Mutations in COX7B cause microphthalmia with linear skin lesions, an unconventional mitochondrial disease. Am J Hum Genet. 2012;91:942-9.

8. Van rahden VA, Fernandez-vizarra E, Alawi M, et al. Mutations in NDUFB11, encoding a complex I component of the mitochondrial respiratory chain, cause microphthalmia with linear skin defects syndrome. Am J Hum Genet. 2015;96:640-50.

9. Wimplinger I, Rauch A, Orth U, Schwarzer U, Trautmann U, Kutsche K. Mother and daughter with a terminal Xp deletion: implication of chromosomal mosaicism and X-inactivation in the high clinical variability of the microphthalmia with linear skin defects (MLS) syndrome. Eur J Med Genet. 2007:50:421-31.

10. Morleo M, Franco B. Microphthalmia with linear skin defects syndrome. Genereviews 2011; Available on: https://www.ncbi.nlm.nih.gov/books/ NBK7041/

11. Temple IK, Hurst JA, Hing S, Butler L, Baraitser M. De novo deletion of Xp22. 2-pter in a female with linear skin lesions of the face and neck, 
microphthalmia, and anterior chamber eye anomalies. J Med Genet. 1990;27:56-8.

12. Kayserili H, Cox TC, Cox LL, et al. Molecular characterisation of a new case of microphthalmia with linear skin defects (MLS). J Med Genet. 2001;38:411-7.

13. Morleo M, Franco B. Dosage compensation of the mammalian X chromosome influences the phenotypic variability of X-linked dominant male-lethal disorders. J Med Genet. 2008;45:401-8.

14. Ogata T, Wakui K, Muroya K, et al. Microphthalmia with linear skin defects syndrome in a mosaic female infant with monosomy for the Xp22 region: molecular analysis of the Xp22 breakpoint and the X-inactivation pattern. Hum Genet. 1998;103:51-6.

Ready to submit your research? Choose BMC and benefit from:

- fast, convenient online submission

- thorough peer review by experienced researchers in your field

- rapid publication on acceptance

- support for research data, including large and complex data types

- gold Open Access which fosters wider collaboration and increased citations

- maximum visibility for your research: over $100 \mathrm{M}$ website views per year

At BMC, research is always in progress.

Learn more biomedcentral.com/submissions 